\title{
INFLUENCE OF RADIATION DAMAGE OF REACTOR INNER COMPONENTS ON THE RESULTS OF INDENTATION TESTS
}

\author{
Jan OndraceK*, Ales Materna \\ Department of Materials, Faculty of Nuclear Sciences and Physical Engineering, Czech Technical University in \\ Prague \\ * corresponding author: jan.ondracek@fjfi.cvut.cz
}

\begin{abstract}
Irradiation of inner components of the WWER reactor type made from austenitic steel $08 \mathrm{Ch} 18 \mathrm{~N} 10 \mathrm{~T}$ alters their material properties and may cause component degradation. Numerical simulations of the indentation test on the non- and neutron-irradiated reactor's inner components were carried out. The aim of this study is to find out whether the indentation test is suitable for assessing the material radiation damage of studied components.
\end{abstract}

KEYWORDS: neutron-irradiated material, hardness, indentation, WWER, FEM.

\section{INTRODUCTION}

The extent of radiation damage of reactor pressure vessel internals (RVI) is a key factor in determining the remaining life span of nuclear power plants. In the WWER reactors, parts of the RVIs are made from Ti stabilized austenitic stainless steel 08Ch18N10T. The overall radiation dose absorbed by the RVIs after 40 years of operation can exceed $40 \mathrm{dpa}$ [1]. The neutron irradiation produces a series of defects in material. With contribution of other factors, such as a higher temperature, dislocation loops, precipitates, cavities and chemical changes on grain boundaries are formed over time. This leads to changes in mechanical properties, namely an increase of yield stress and hardness and decrease of ductility. It is important to assess how the material properties change in dependence on the overall radiation dose. Since the irradiated material is scarce, hardness testing, which needs only little testing material compared to other testing methods, might be used to estimate material properties of neutron-irradiated materials.

Numerical simulations of hardness testing (HT) of non- and neutron-irradiated materials were made in this study in order to decide whether the HT can reliably estimate the radiation damage of the studied material. The material used in this analysis is the $08 \mathrm{Ch} 18 \mathrm{~N} 10 \mathrm{~T}$ austenitic stainless steel, analogous to ASTM A321 stainless steel. Two experimental datasets of tensile tests taken from the literature were used in this study. The material for specimens in the first dataset [1, 2] was withdrawn from the decommissioned WWER 440 NPP in Greifswald. Tensile tests of non- and neutron-irradiated samples with dose of 5.2 dpa conducted in $20^{\circ} \mathrm{C}$ air environment from [1, 2] were also accompanied by micro hardness tests. The specimens used in the second dataset 3 were cut out of a hot-rolled plate, then annealed at $1050^{\circ} \mathrm{C}$ for $2 \mathrm{~h}$ and cooled in the air. The irradiation of these specimens was carried out in BOR-60 reactor in SSC RIAR. The irradiation temperature was between $320^{\circ}$ and $340{ }^{\circ} \mathrm{C}$. The neutron-irradiation of the samples from the second dataset was 7 and 40-43 dpa.

Elastic-plastic behavior of the material was evaluated based on the tensile tests and then fed into numerical models of micro hardness testing. 2D and 3D FEM models were used in order to obtain not only micro hardness results but also to evaluate surface of the material near the indent for a possible occurrence of sink-ins or pile-ups.

\section{MATERIAL DATA}

Five tensile tests with four different neutron doses (0, 5.2, 7 and 40-43 dpa) taken from [1] and [3] were used to obtain yield strength and a plastic response of the material. The resulting experimental plastic responses as well as the yield stresses are plotted in Figure 1 . The non-irradiated samples show a hardening effect and their hardening curve can be fitted with a Hollomon's equation. The sample irradiated to $7 \mathrm{dpa}$ also shows some hardening effect but the hardening curve could not be fitted with a power law relation. Therefore, no hardening was used in the range of plastic deformation between 0.2 and 1 . In case of samples irradiated to 5.2 and 40-43 dpa no hardening was observed in the tensile tests, so an ideally plastic material response was used in the simulations. Elastic constants of the 08Ch18N10T stainless steel are following: Young modulus equals $200 \mathrm{GPa}$ and Poisson ratio is 0.3 .

\section{NumERICAL MODELING}

In order to compute micro hardness of the nonirradiated and irradiated material using a FEM model, a two-dimensional axisymmetric mesh as well as threedimensional mesh was used. The real shape of the indenter in case of the two-dimensional mesh is represented by an equivalent cone with cone apex semiangle of $70.3^{\circ}$. The cone indenter of this shape has the same projected contact area per penetration depth as 


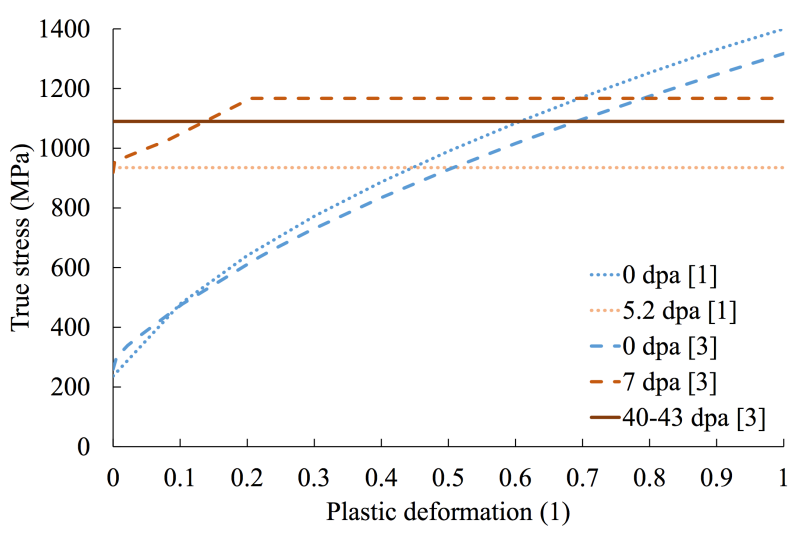

FIGURE 1. Plastic response of studied neutron radiation damages

the Vickers indenter. This indenter is modeled as a perfectly rigid body with no friction with the sample and its movement is controlled by force with a maximum value $1 \mathrm{~N}$. The indenter penetrates the mesh in the contact area. The size of the 4-node quadrilateral elements in the contact area is $0.5 \times 0.2 \mu \mathrm{m}$ and the size of the rectangular contact area is $40 \times 40 \mu \mathrm{m}$. The rest of the two-dimensional mesh is auto-meshed with three node triangular elements. Their size gradually grows with their distance from the contact area. The whole set-up of the 2D model can be seen in Figure 2 . Boundary conditions are following: All nodes on the bottom edge of the model are fixed in the direction perpendicular to the direction of loading in order to achieve a rotational symmetry of the model and all nodes on the right-most edge are fixed in the direction of loading.

The 3D mesh allows modeling a real shape of the Vickers four-sided pyramid indenter. Therefore, the true imprint as well as the pile-up profile can be studied. The three-dimensional mesh consists of 180000 8-node hexahedral elements. Only one fourth of the sample surface is modeled in order to save computing time. The indentation region with the smallest elements has size of $30 \times 30 \times 10 \mu \mathrm{m}(10 \mu \mathrm{m}$ in the direction of loading). The element size in this region is $0.5 \times 0.5 \times 1 \mu \mathrm{m}$ ( $1 \mu \mathrm{m}$ in the direction of loading). The three-dimensional mesh is shown in Figure 3 All nodes at the symmetry boundary planes have fixed displacements in the direction perpendicular to the plane. The indenter is modeled by a rigid plane. The movement of the indenter plane is driven by displacement until the maximum loading force at the indenter tip $1 \mathrm{~N}$ is reached. This procedure provides a sufficient numerical stability.

The plastic response of the material was modeled using isotropic hardening with von Mises yield condition. Plastic responses used in the simulations are shown in Figure 1. All the simulations were run in the large-strain regime.

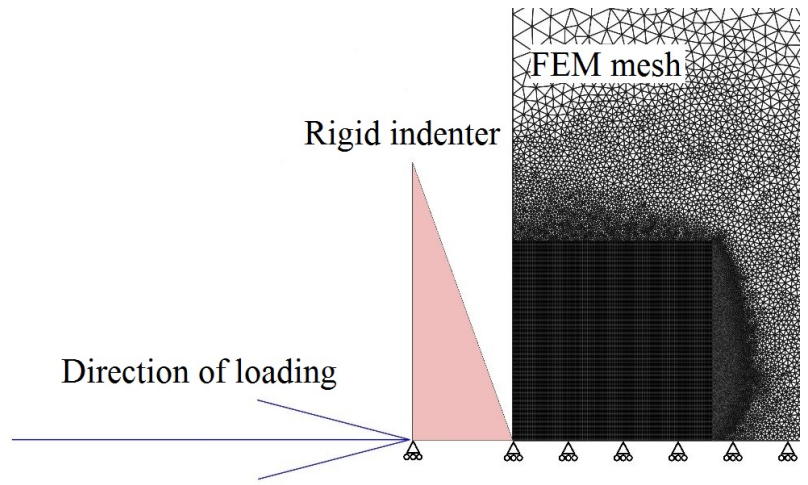

Figure 2. Two-dimensional FEM model of the indentation test.

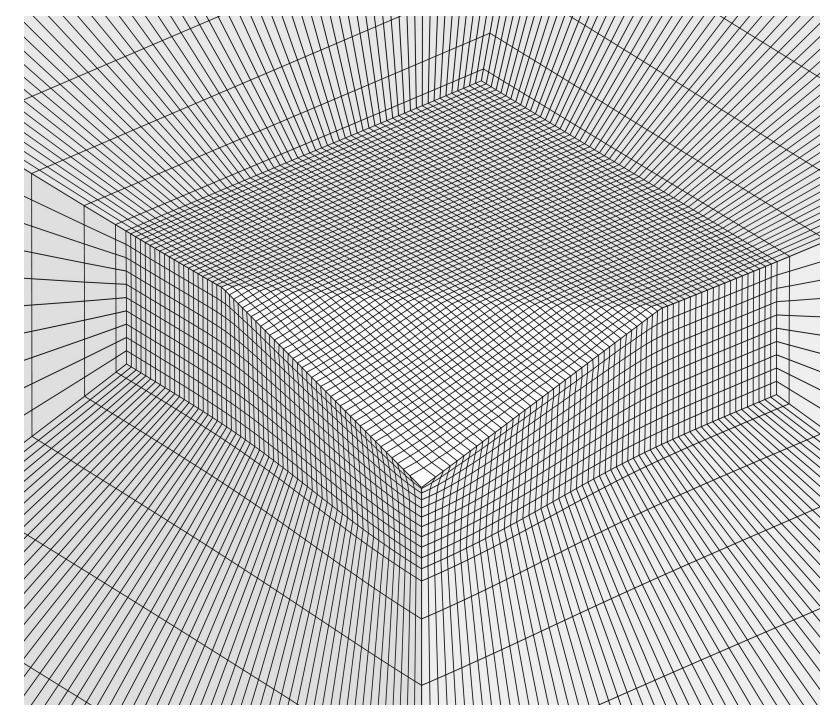

FiguRE 3. Three-dimensional mesh of a material surface with an indentation imprint

\section{Results And Discussion}

The results compose of $2 \mathrm{D}$ and $3 \mathrm{D}$ indentation test simulations. Five computations with different neutronirradiation damage were performed using MSC.MARC software for FEM analyses. The mechanical properties data were taken from two different datasets. The corresponding dataset is labeled by its reference number in the results. Indentation load - penetration depth curves and hardnesses can be evaluated using either two- or three-dimensional mesh. The differences are however within a numerical error range, so only the results for $2 \mathrm{D}$ mesh are shown. Three-dimensional computations used in this study took approximately $10 \mathrm{x}$ times longer to compute than the two-dimensional ones, but they provide a better insight into the expected indent shape and surface profiles.

Indentation load - penetration depth curves are shown in Figure 4. The maximum penetration depth reaches a little over $5 \mu \mathrm{m}$ for both non-irradiated specimens. The specimen from the dataset 1 has higher penetration depth than the one from [3] and their difference is $0.135 \mu \mathrm{m}$. The neutron-irradiated spec- 


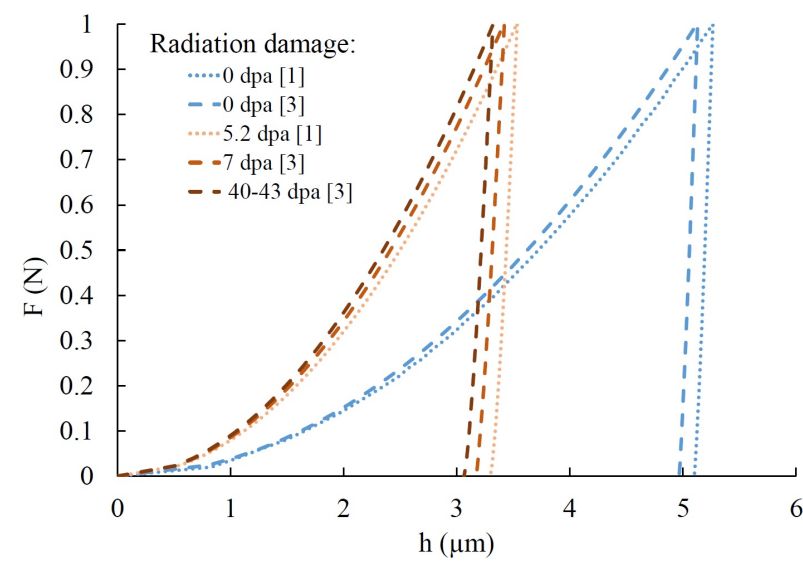

FiguRE 4. Simulated indentation curves using a 2D model for non- and neutron-irradiated material, mechanical properties determined at room temperature

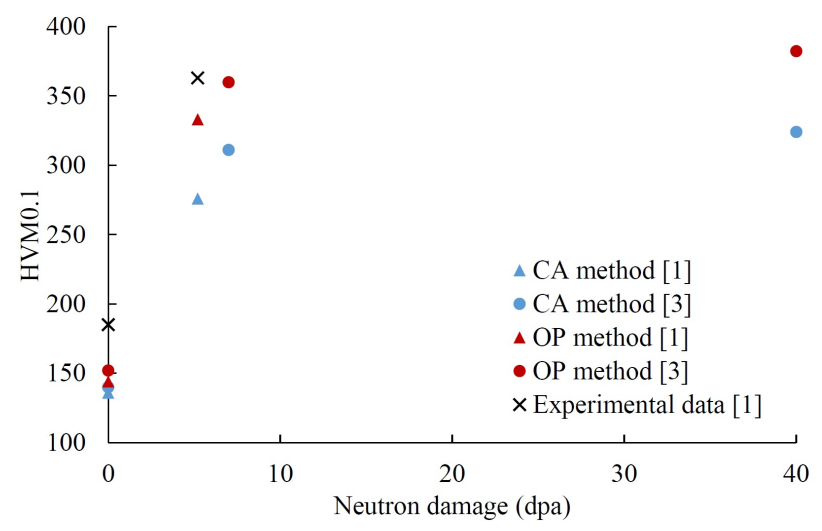

FiguRE 5. Dependence of Vickers hardness on neutron damage for different hardness evaluation methods: contact area (CA), Oliver-Pharr (OP) 4 method

imens are clearly harder and their maximum penetration depth is within a range of 3.32 and $3.53 \mu \mathrm{m}$. The curves are ordered by their radiation damage with the most irradiated specimen having the smallest maximum penetration depth.

Analogous results are shown in Figure 5. Vickers hardness is evaluated using a direct contact area method (CA) and the Oliver-Pharr method [4, 5]. In case of the $\mathrm{CA}$ method, the radius of the circular projected contact area at maximum load is read from the results. It is easy to recognize the furthest node in contact with the indenter at the maximum load. Since the displacement in the direction of loading after a complete unloading of this node is minimal, the diameter of the circular contact area is evaluated based on this node. Although the results of hardness are shown in the units of Vickers hardness for the sakes of comparing to experimental data [1, 2, it is interesting to obtain hardnesses using the Oliver-Pharr method as well. The computed unloading force-penetration depth curves (Fig. 4) were used to obtain hardness by the OP method.

The computed hardnesses from dataset [1] can be compared to experimental data. The computed hard-

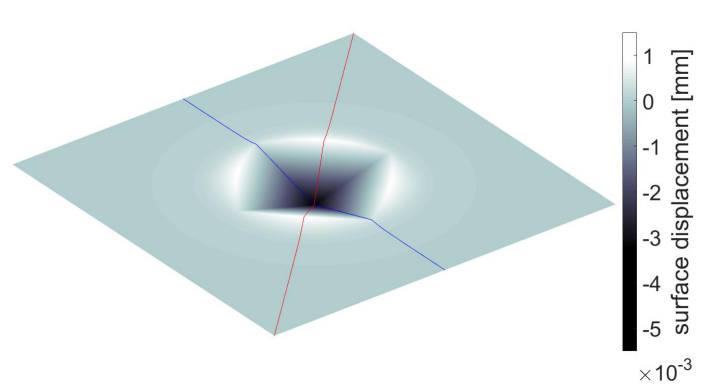

FiguRE 6. Surface profile cross-sections; Face profile (red), diagonal profile (blue)

ness evaluated by the CA method of non-irradiated and neutron-irradiated material with dose $5.2 \mathrm{dpa}$ is approx. $25 \%$ smaller than experimentally assessed. It can be also seen than the computed hardness rises with rising neutron damage, although the increase in hardness between 7 and 40-43 dpa is insignificant. The resulting computed hardness also depends on a method used for evaluation.

The OP method gives higher hardnesses for all the specimens. The difference in hardness for nonirradiated specimens is approximately $6 \%$. The methods differ by approx. 16\% from each other for neutronirradiated material. Such a large difference can be explained by the fact that the Oliver-Pharr method uses only penetration depth curves as an input for estimating the contact area using only an elastic contact solution (Fig. 4). A little or no hardening material is used for simulations of neutron-irradiated specimens (see Figure 1). Therefore, pile-ups form in the vicinity of the indent and the estimated contact area differs greatly from the one coming from an elastic-plastic calculation. The non-irradiated strainhardening material exhibits smaller pile-ups compared to the neutron-irradiated one.

Created pile-ups are clearly visible in Figure 7 showing the surface profiles for all the specimens. The surface profiles are plotted in the diagonal and face cross-section of the three-dimensional indent (as can be seen in 60 in colors, the dashed line shows a surface profile computed using a two-dimensional mesh. The 2D profile lies between the face and diagonal surface profiles as expected. The maximum pile-up height occurs at the three-dimensional indent face. The nonirradiated specimens (Fig. 7A, C) have much smaller pile-ups than the irradiated ones because their material strain hardens. The biggest pile-up was calculated in Fig. 7B because the yield strength of its material is smaller compared to more irradiated Fig. $7 \mathrm{E}$ and no strain hardening was used for both computations. The overall visualizations of the indents in surrounding material can be seen in Figure 8

\section{Conclusions}

Two- and three-dimensional FEM models of indentation test were created in order to assess hardness of 


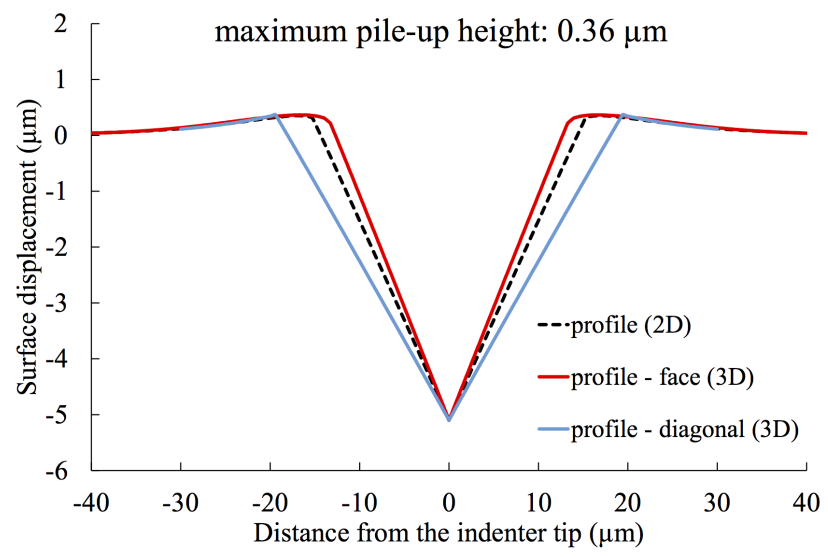

(A) . 0 dpa [1]

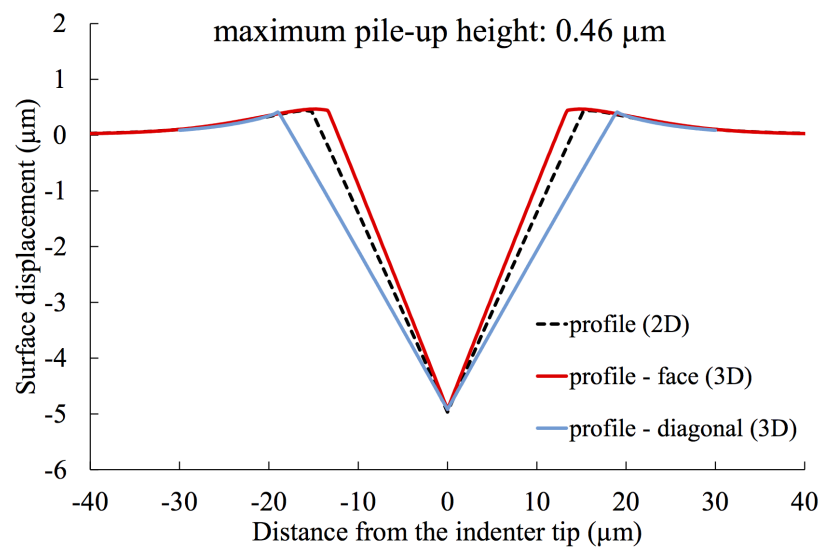

(C) . 0 dpa 3

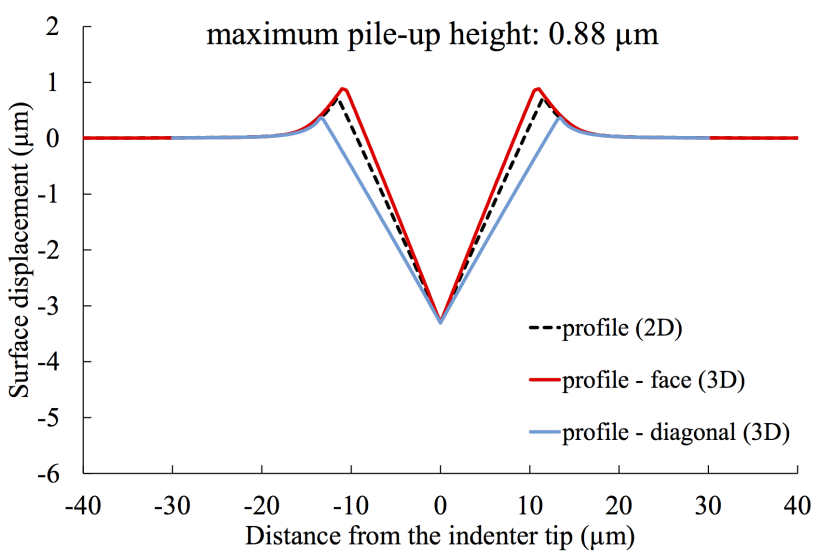

(B) . $5.2 \mathrm{dpa}[1]$

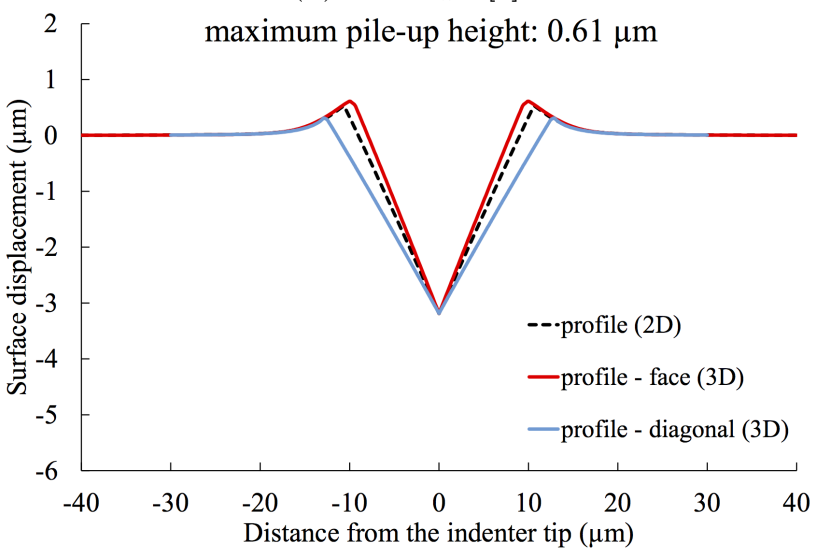

(D) $.7 \mathrm{dpa}[3$

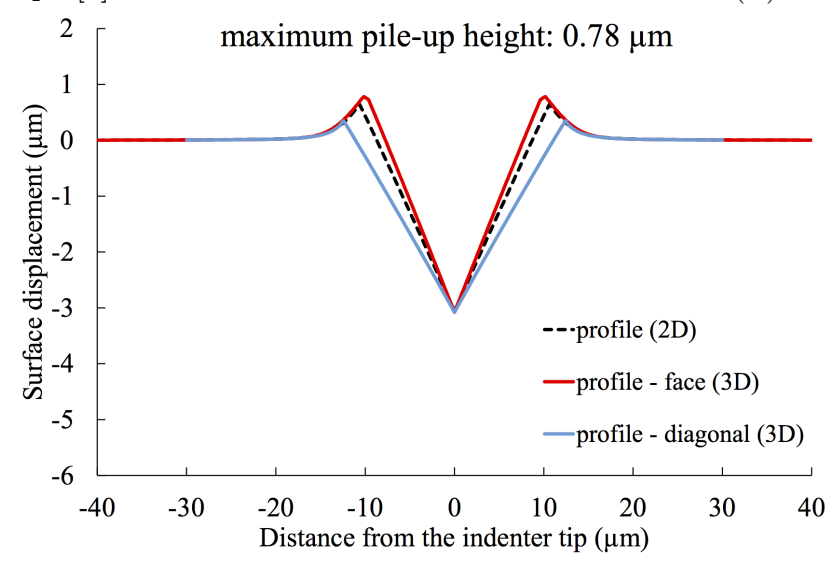

(E) . 40-43 dpa [3]

Figure 7. Computed surface displacement profiles; non-irradiated samples A and C, neutron irradiated samples B, $\mathrm{D}$ and $\mathrm{E}$; , dataset [1] A and B, dataset [3] C, D and E; depicted surface area 40x40 $\mu \mathrm{m}$ 


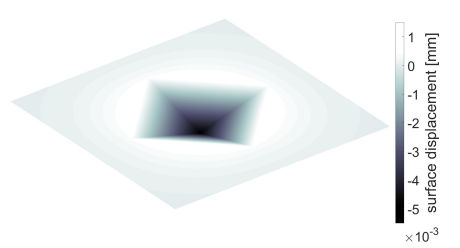

(A) .0 dpa $\underline{3}$

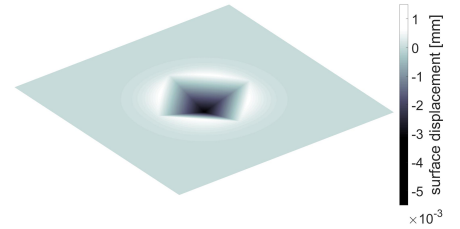

(B) .7 dpa $\underline{3}$

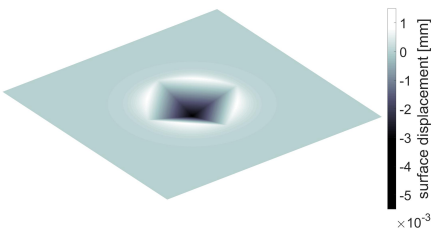

(C) . 40-43 dpa 3

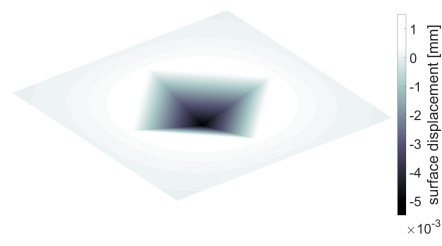

(D) $.0 \mathrm{dpa}[1$

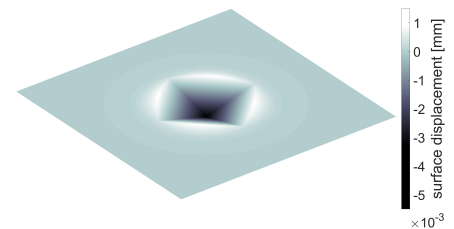

(E) . 5.2 dpa 1$]$

Figure 8. Computed indents; non-irradiated samples A and D, neutron-irradiated samples B, C and E; dataset [3] A, B and C, dataset [1 D and E; depicted surface area 30x30 $\mu \mathrm{m}$

neutron-irradiated stainless steel 08Ch18N10T. The two-dimensional model used an equivalent cone indenter with a cone apex semi-angle of $70^{\circ}$ to simulate Vickers indenter shape and in the three-dimensional model the Vickers 4-sided pyramid was directly modeled. Necessary material input data was obtained using tensile tests taken from the literature. A part of the the tensile test of neutron-irradiated specimens was accompanied by experimentally measured hardnesses. The following conclusions can be made based on the work presented here:

(1.) Both two- and three-dimensional models predict the same hardness. The 3D model in addition is able to compute surface displacement profiles for face and diagonal cross-sections of the indent as well as the shape of the indent including possible pile-ups in its vicinity.

(2.) The numerical models predict higher hardnesses for neutron-irradiated material which is in agreement with experiments.

(3.) The Oliver-Pharr method overestimates hardness of neutron-irradiated material because pile-ups build up in the vicinity of the impression.

\section{ACKNOWLEDGEMENTS}

This work was supported by the Technology Agency of the Czech Republic, project No. TH02020565.

\section{REFERENCES}

[1] A. Hojna, M. Ernestova, O. Hietanen, et al. Irradiation assisted stress corrosion cracking of austenitic stainless steel WWER reactor core internals. In G. I. J. T. Busby, P. L. Andresen (eds.), 15th International Conference on Environmental Degradation of Materials in Nuclear Power Systems Water Reactors. John Wiley and Sons, Inc., Hoboken, New Jersey, Canada, 2011. DOI:10.1002/9781118456835.ch131

[2] H. K. Namburi, A. Hojna, Z. Fencl. Effect of tensile strain on microstructure of irradiated core internal material. In International Conference Nuclear Energy for New Europe, Portoroz, Slovenia. 2015.

[3] A. Sorokin, B. Margolin, I. Kursevich, et al. Effect of neutron irradiation on tensile properties of materials for pressure vessel internals of WWER type reactors. $J$ Nucl Mater 444:373-384, 2014. DOI:10.1016/j.jnucmat.2013.10.016

[4] W. Oliver, G. M. Pharr. An improved technique for determining hardness and elastic modulus using load and displacement sensing indentation experiments. J Mater Res 7:1564-1583, 1992. DOI:10.1557/JMR.1992.1564.

[5] W. Oliver, G. M. Pharr. Measurement of hardness and elastic modulus by instrumented indentation: Advances in understanding and refinements to methodology. $J$ Mater Res 19:3-24, 2004. DOI:10.1557/jmr.2004.19.1.3. 